\title{
MAGNETOELECTRIC EFFECT IN FERROMAGNETIC-SEMICONDUCTOR LAYERED COMPOSITE STRUCTURES
}

\author{
Leonid Fetisov ${ }^{1,}{ }^{*}$, Dmitri Chashin ${ }^{1}$, Dmitri Saveliev ${ }^{1}$, Daria Plekhanova ${ }^{1}$, Ludmila Makarova ${ }^{2}$, and Alexandr Stognii ${ }^{3}$ \\ ${ }^{1}$ Moscow Technological University, Moscow, Russia \\ ${ }^{2}$ Moscow State University, Moscow, Russia \\ ${ }^{3}$ Scientific and Practical Materials Research Centre of NAS of Belarus, Minsk, Belarus
}

\begin{abstract}
The results of magnetoelectric effect experimental studies in two different structures based on piezoelectric semiconductor gallium arsenide are presented. The monolithic structure consisted of a gallium arsenide substrate with deposited nickel layer (GaAs-Ni), and the composite structure contained a semiconductor substrate with an amorphous magnetic alloy (GaAs-Metglas) ribbon glued on one side. A quality factor $Q \approx 23500$ and magnetoelectric coefficient of $316 \mathrm{~V} / \mathrm{Oe} \cdot \mathrm{cm}$ were achieved at the frequency of planar acoustic oscillations for GaAs-Ni structure at room temperature.
\end{abstract}

\section{Introduction}

Magnetoelectric (ME) effect manifests itself as polarization of the sample in the external magnetic field and observed in layered composite magnetostrictive-piezoelectric structures due to mechanical coupling between the layers [1]. Magnetic materials with large magnetostriction $\lambda$ (Ni, Co, alloys FeCo, FeGa, terfenol, FeSiCB etc.) are used as magnetic layers in such structures. Most commonly used piezoelectric materials are various lead-based ceramics (PZT, PMN-PT), which posses good electric and piezoelectric properties.

There are number of other materials with high piezomodule to dielectric permittivity ratio $d_{31} / \varepsilon$, that is required for effective ME interaction, which are used in such composite structures along with ceramic piezoelectric materials: piezoelectric single crystals (langatate and quartz) [2] and flexible piezopolymers, like PVDF [3].

It is also known that some of semiconductor materials demonstrate piezoeffect, such as GaAs, ZnS, and CdS. Piezoelectric moduli of these materials lay in the range $2 \cdot 10^{-12}-14 \cdot 10^{-}$ $12 \mathrm{C} / \mathrm{N}$, and resistivity exceeds $10^{6} \Omega \cdot \mathrm{cm}$. Magnetoelectric effect was recently experimentally discovered in composite structure based on GaAs semiconductor substrate [4-5]. Application of semiconductor materials in such composite structures will expand the functionality of $\mathrm{ME}$ devices. Standard planar technologies can be used for ME semiconductor-based devices production, which, for sure, will facilitate the integration of future magnetoelectric devices into modern electronic components.

In this paper authors investigated frequency and field characteristics of ME effect in composite structures based on semiconductor gallium arsenide substrate with most commonly used magnetostrictive materials such as nickel and amorphous magnetic alloy.

\section{Structures and set-up}

The measurements were carried out on two samples: monolithic structure GaAs-Ni and composite structure GaAs-Metglas. Commercially available semiconductor GaAs plates of lateral dimensions $13.5 \mathrm{~mm} \times 5 \mathrm{~mm}$ and thickness $0.4 \mathrm{~mm}$, cut along natural edges of the crystal with orientation (100) were used for structures fabrication. Gold electrodes with $\sim 1 \mu \mathrm{m}$ thickness were deposited on the surface of the plates. Monolithic structure GaAs-Ni was fabricated by the ion beam sputtering method by depositing $\sim 1.4 \mu \mathrm{m}$ thickness $\mathrm{Ni}$ 
layer on one side of the GaAs substrate [6]. Electronic image of this structure was obtained at FIB station FIE 450 in the Nanocentre of MIREA (see Fig. 1). The structure is characterized by the clear plane-parallel filmsubstrate and film-surface interface boundaries. The surface of the nickel film is uniform and the dimensions of the inhomogeneity's are close to the monomodal distribution at the submicron level. The composite structure was fabricated by gluing a $\sim 30 \mu \mathrm{m}$ thick ribbon of an amorphous alloy FeBSiC (Metglas) to one side of the GaAs plate. The structures were fastened with thin wires in the center in such a way that only longitudinal acoustic vibrations could be excited.

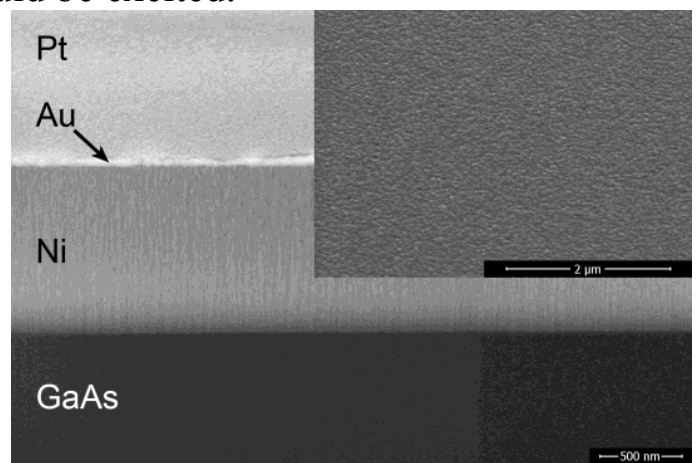

Fig. 1. Electronic image of the monolithic GaAs-Ni structure.

The samples were placed into external permanent magnetic field $H$ and excited by the alternating magnetic field $h \cos (2 \pi f t)$, both directed parallel to the long side of the structure. The dependencies of the voltage $U$ generated across the electrodes of the GaAs layer were recorded when the field $H$ was varied in the range $0-120 \mathrm{Oe}$, frequency $f$ of the ac field was varied in the range $0-200 \mathrm{kHz}$, and amplitude $h$ of the ac field changed from 0 to 10 Oe. The coefficient of ME interaction was calculated using the formula $\alpha=U /(h \cdot b)$ where $U$ is the voltage amplitude, $h$ is the ac field amplitude, and $b$ is the thickness of the semiconductor layer. The dielectric properties were measured with RLC meter Actacom AM3026. The magnetic properties of a monolithic sample were measured using a LakeShore 7404 vibrating sample magnetometer when the structure was magnetized along its long side.

\section{Results and discussion}

Frequency dependencies of the dielectric permittivity and resistance for GaAs plates are shown in Fig. 2. It is seen that the resistance of the semiconductor decreases by a factor of 50 , from 7.2 $\mathrm{M} \Omega$ to $0.145 \mathrm{M} \Omega$, as the frequency increases. The dielectric constant $\varepsilon$ of the semiconductor, calculated using the measured capacitance of the structure, decreases sharply with increasing frequency from $\sim 85$ at a frequency $f=5 \mathrm{~Hz}$ to $\sim 16$ at frequencies $f>1$ $\mathrm{kHz}$.

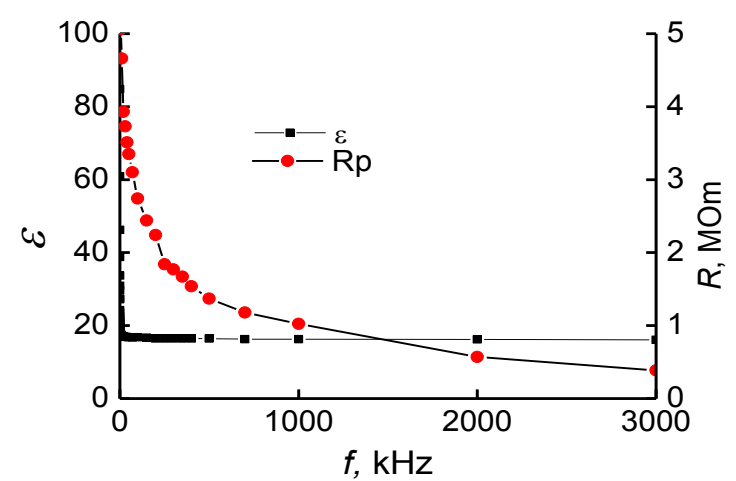

Fig. 2. Frequency dependencies of the dielectric permittivity and resistance for GaAs plate.

Hysteresis of the magnetic moment $M$ of the monolithic GaAs-Ni structure is shown in Fig. 3. As it follows from the figure, magnetic layer of the structure saturates in the fields $H_{\mathrm{s}} \sim 60 \mathrm{Oe}$. The coercive field of the structure is $H_{\mathrm{c}} \sim 11 \mathrm{Oe}$.

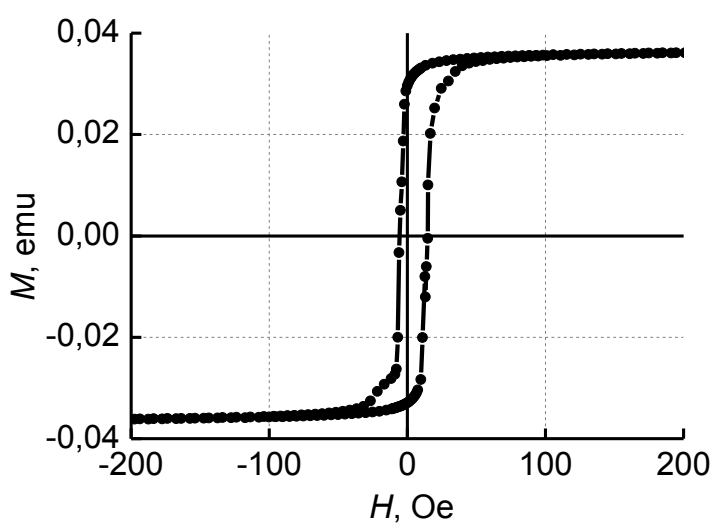

Fig. 3. Hysteresis loop for GaAs-Ni sample.

The dependencies of ME voltage, generated by the structures, on the excitation field frequency $f$ measured during the experiment are shown in Fig. 4. Measurements were carried out at $H=20 \mathrm{Oe}, h=0.01$ Oe for the GaAs-Ni sample and at $H=25 \mathrm{Oe}, h=0.01 \mathrm{Oe}$ 
for the GaAs-Metglas sample. The monolithic structure GaAs-Ni demonstrates maximum of ME voltage $U_{1}=127 \mathrm{mV}$ at the planar resonance frequency $f_{1}=155.17 \mathrm{kHz}$. The quality factor of the monolithic structure is $Q_{1}$ $\approx 23.5 \cdot 10^{3}$. Magnetoelectric coefficient at resonance frequency is $\alpha_{1} \approx 316 \mathrm{~V} / \mathrm{Oe} \cdot \mathrm{cm}$. The composite GaAs-Metglas structure generates ME voltage with maximum amplitude $U_{2}=$ $63.9 \mathrm{mV}$ at the planar resonance frequency $f_{2}=$ $174.34 \mathrm{kHz}$. The quality factor of the composite structure $Q_{2} \approx 1850$ is an order of magnitude smaller than that one of monolithic structure. Magnetoelectric coefficient for the composite structure is $\alpha_{2} \approx 160 \mathrm{~V} / \mathrm{Oe} \cdot \mathrm{cm}$ at resonance.

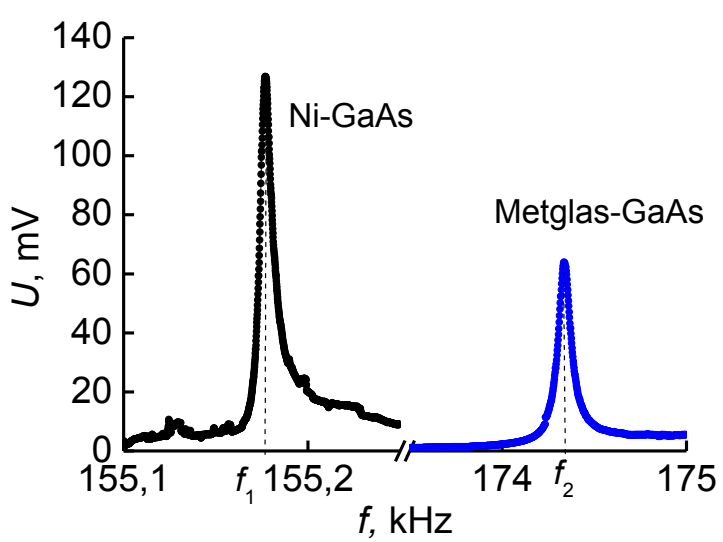

Fig. 4. Frequency dependencies of the ME voltage for GaAs$\mathrm{Ni}$ and GaAs-Metglas samples.

Thus, the efficiency of the ME interaction in the monolithic structure GaAs-Ni under resonance turned out to be $\sim 2$ times higher than that one of the composite GaAs-Metglas structure, despite the fact that the thickness of the nickel layer is much smaller than the thickness of the Metglas layer. Apparently, the sputtering provides better mechanical contact between the layers than the gluing. Due to low thickness of the Ni layer the quality factor of the monolithic structure turned out to be $\sim 13$ times higher, which also leads to an increase in the ME interaction efficiency.

The dependencies of the ME voltage, quality factor and frequency of the resonance on the constant magnetic field for both structures are shown in Figs. 5 and 6. Measurements of the ME voltages and quality factors were carried out at frequencies $f_{1}$ and $f_{2}$ under excitation of the structure by ac magnetic field with amplitude $h=0.01$ Oe. The ME voltage $U(\mathrm{H})$ for the monolithic sample with $\mathrm{Ni}$ layer (see Fig. 5a) reaches maximum in small fields $H \sim 3$ Oe and voltage vs. magnetic field dependence demonstrates hysteretic behavior while the field decreases. The ME voltage vs. magnetic field dependence for the composite structure with Metglas layer (see Fig. 6a) demonstrates classical shape with maximum value in the field $H \sim 28 \mathrm{Oe}$, which corresponds to the maximum of the piezomagnetic coefficient for the magnetostrictive layer.
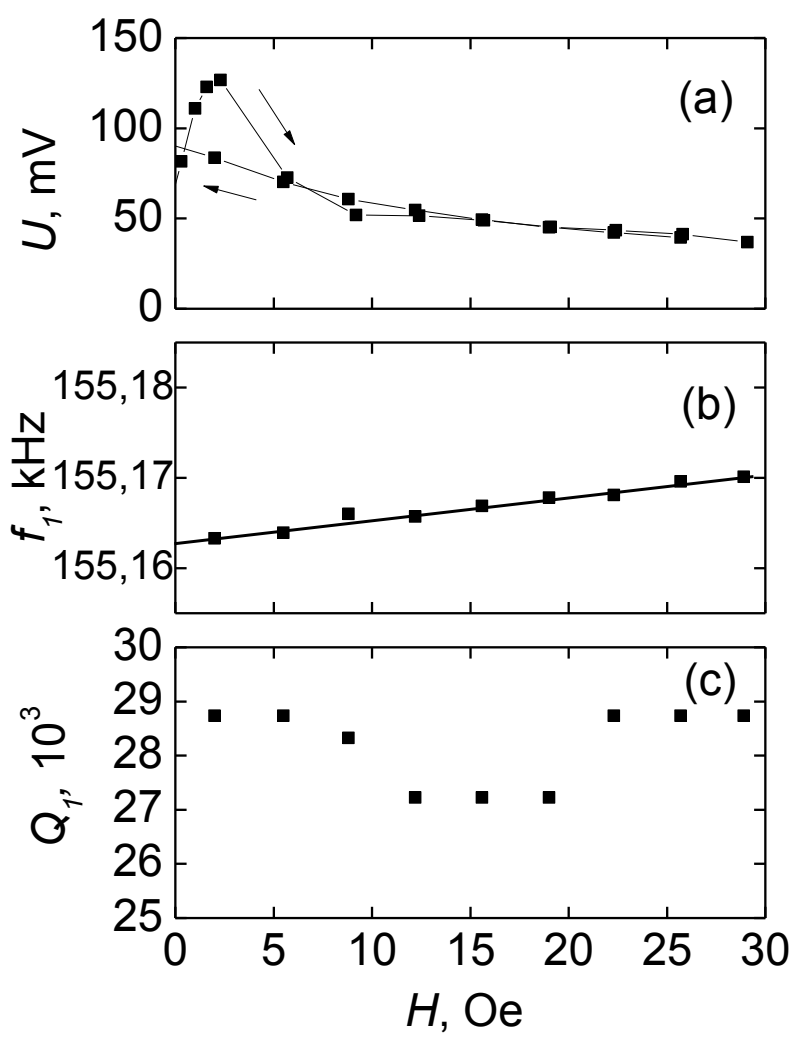

Fig. 5. Dependencies of the ME voltage $U_{1}$, resonance frequency $f_{1}$ and quality factor $Q_{1}$ on the field $H$ for monolithic structure GaAs-Ni.

Resonance frequencies for both structures (see Fig. 5b and Fig. 6b) changed no more than on $0.005 \%$ with the permanent field increase. However, due to the bigger quality factor of the monolithic structure, even that small shift of the frequency results in significant change of the ME voltage measured near the resonance. From Fig. 5c and $6 \mathrm{c}$ it follows that when the constant field $H$ is increased the acoustic quality factor for the monolithic structure changes no more than on $6 \%$, whereas the 
quality factor of the composite structure changes on $\sim 20 \%$. Resonance frequency and quality factor dependences on the magnetic field may be due to the Young's modulus and dielectric losses magnetic field dependencies.
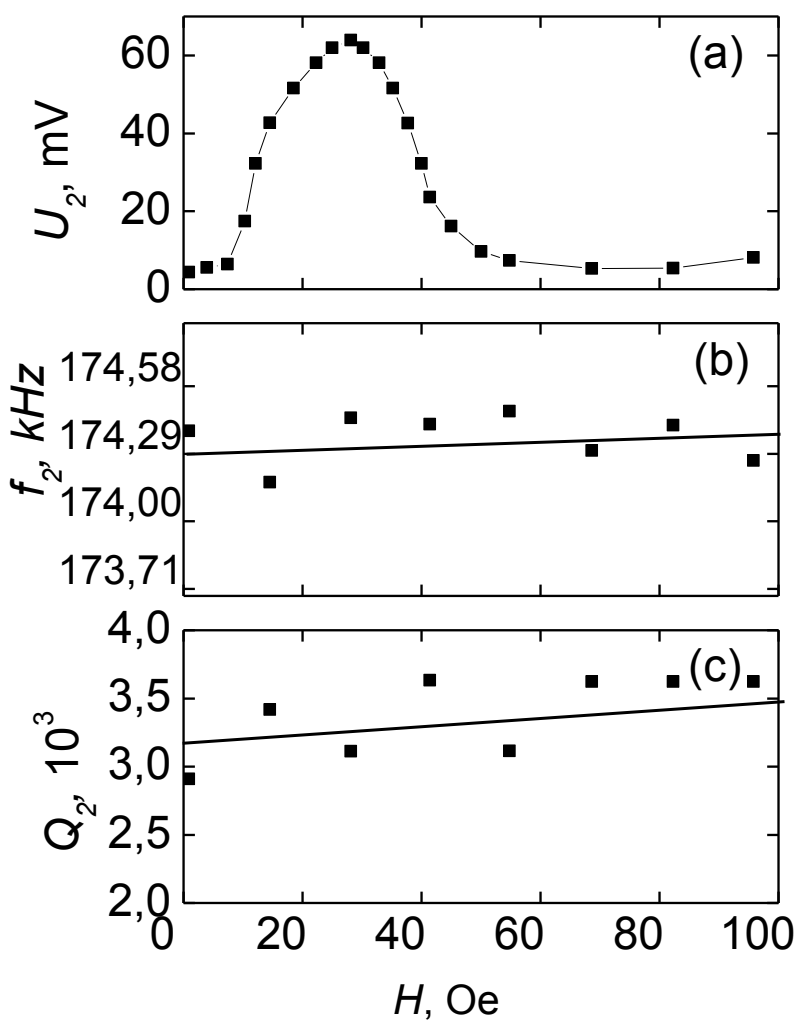

Fig. 6. Dependencies of the ME voltage $U_{2}$, resonance frequency $f_{2}$ and quality factor $Q_{2}$ on the field $H$ for the composite structure GaAs-Metglas.

Thus, the resonant ME effect in composite structures based on the piezoelectric GaAs semiconductor was investigated. The ME coefficient at the acoustic resonance frequency was $\alpha_{1} \approx 316 \mathrm{~V} /$ Oe $\cdot \mathrm{cm}$ for the monolithic GaAs-Ni structure and $\alpha_{2} \approx 160 \mathrm{~V} / \mathrm{Oe} \cdot \mathrm{cm}$ for the composite GaAs-Metglas structure. The efficiency of ME interaction in a monolithic sample is higher due to better mechanical coupling between layers and smaller acoustic losses in a thin Ni layer.

The work was supported by the Russian Science Foundation (agreement 17-12-01435).

\section{References}

1. C.-W. Nan, M.I. Bichurin, and S. Dong, J. Appl. Phys. 103031101 (2008)
2. G. Sreenivasulu, L.Y. Fetisov, G. Srinivasan, and Y.K. Fetisov, Appl. Phys. Let. V. 100 №. 052901 (2012)

3. P. Martins and S. Lanceros-Méndez, Adv. Funct. Mater. 233371 (2013)

4. D.A. Filippov, T.O. Firsova, V.M. Laletin, N.N. Poddubnaya, Письма в ЖТФ Vol. 43, №. 6, pp. 7277. (2017)

5. V.M. Laletin, A.I. Stognii, N.N. Novitskii, N.N. Poddubnaya, Письма в ЖТФ Vol. 40, №. 21, pp. 71-78 (2014)

6. A. I. Stognij, N. N. Novitskii, V. A. Ketsko, S. A. Sharko, N. N. Poddubnaya, V. M. Laletin, A. V. Bespalov, O. L. Golikova, M. N. Smirnova, L. Yu. Fetisov, and A. O. Titova, Inorganic Materials Vol. 52 № 10 pp. 1070-1076 (2016) 